\section{(6) OPEN ACCESS}

\title{
Pretreatment multi-biomarker disease activity score and radiographic progression in early RA: results from the SWEFOT trial
}

\author{
Karen Hambardzumyan, ${ }^{1}$ Rebecca Bolce, ${ }^{2}$ Saedis Saevarsdottir, ${ }^{1,3}$ \\ Scott E Cruickshank, ${ }^{4}$ Eric H Sasso, ${ }^{2}$ David Chernoff, ${ }^{2}$ Kristina Forslind, ${ }^{5,6}$ \\ Ingemar F Petersson, ${ }^{5,7}$ Pierre Geborek, ${ }^{5}$ Ronald $\mathrm{F}$ van Vollenhoven ${ }^{1}$
}

\section{Handling editor Tore K Kvien \\ - Additional material is published online only. To view please visit the journal online (http://dx.doi.org/10.1136/ annrheumdis-2013-204986).}

For numbered affiliations see end of article.

\section{Correspondence to Karen Hambardzumyan, Department of Medicine, Clinical Therapy Research, Inflammatory Diseases (ClinTRID), Karolinska Institute, D1:00 Karolinska University Hospital, Solna, Stockholm 17176, Sweden;} karen.hambardzumyan@ki.se

Received 27 November 2013 Revised 4 April 2014 Accepted 13 April 2014 Published Online First 8 May 2014

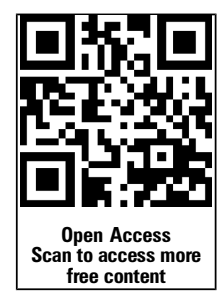

\section{ABSTRACT}

Objectives Prediction of radiographic progression (RP) in early rheumatoid arthritis (eRA) would be very useful for optimal choice among available therapies. We evaluated a multi-biomarker disease activity (MBDA) score, based on 12 serum biomarkers as a baseline predictor for 1-year RP in eRA.

Methods Baseline disease activity score based on erythrocyte sedimentation rate (DAS28-ESR), disease activity score based on C-reactive protein (DAS28-CRP), CRP, MBDA scores and DAS28-ESR at 3 months were analysed for 235 patients with eRA from the Swedish Farmacotherapy (SWEFOT) clinical trial. RP was defined as an increase in the Van der Heijde-modified Sharp score by more than five points over 1 year. Associations between baseline disease activity measures, the MBDA score, and 1-year RP were evaluated using univariate and multivariate logistic regression, adjusted for potential confounders.

Results Among 235 patients with eRA, 5 had low and 29 moderate MBDA scores at baseline. None of the former and only one of the latter group (3.4\%) had RP during 1 year, while the proportion of patients with RP among those with high MBDA score was 20.9\% $(p=0.021)$. Among patients with low/moderate CRP, moderate DAS28-CRP or moderate DAS28-ESR at baseline, progression occurred in 14\%, 15\%, 14\% and $15 \%$, respectively. MBDA score was an independent predictor of RP as a continuous (OR=1.05, 95\% Cl 1.02 to 1.08 ) and dichotomised variable (high versus low/moderate, $\mathrm{OR}=3.86,95 \% \mathrm{Cl} 1.04$ to 14.26). Conclusions In patients with eRA, the MBDA score at baseline was a strong independent predictor of 1-year RP. These results suggest that when choosing initial treatment in eRA the MBDA test may be clinically useful to identify a subgroup of patients at low risk of RP.

Trial registration number WHO database at the Karolinska Institute: CT20080004; and clinicaltrials.gov: NCT00764725.

\section{INTRODUCTION}

The course of rheumatoid arthritis (RA) can vary from mild and non-destructive to severe and rapidly destructive. ${ }^{12}$ Although some clinical parameters at diagnosis, including inflammatory markers, baseline erosions, smoking, and in some studies, auto-antibody status, have been shown to be associated with the risk of radiographic progression (RP), ${ }^{3-14}$ they have limited predictive power on an individual basis. Therefore, identification of new predictors would be beneficial for establishing the prognosis at an early stage and for optimally choosing therapy.

Various serum biomarkers have been studied as predictors of RP. For example, bone and cartilage metabolism turnover are found to be associated with RP of joint damage in patients with RA, ${ }^{15-17}$ whereas high leptin and eotaxin levels, although being pro-inflammatory, are associated with better radiographic outcomes. ${ }^{18} 19$ To date, no single biomarker has proven to be highly reliable for predicting $\mathrm{RP}^{20}{ }^{21}$ Therefore, the use of combinations of biomarkers may be a more promising approach.

The multiple-biomarker disease activity (MBDA; Crescendo Bioscience Inc, South San Francisco, California, USA) score (range from 1 to 100) is based on serum levels of several biomarkers. The development of the MBDA score started with screening 396 candidate biomarkers and ended up with 12 that were combined into a score and shown to correlate well with disease activity. ${ }^{22-24}$ This test is validated for clinical use in the USA as a disease activity marker in RA. Its value as a predictor of clinical and radiographic outcomes is currently the subject of several studies. Bakker et $a l^{25}$ showed in the CAMERA study that the MBDA score correlated significantly $(\mathrm{r}=0.72 ; \mathrm{p}<0.001)$ with disease activity score based on C-reactive protein (DAS28-CRP). Hirata et $a l^{26}$ observed an association of the MBDA score and its 1-year change with different clinical outcomes and Van der Helm-Van Mil et al ${ }^{23}$ demonstrated that remission based on the MBDA score was associated with limited RP in patients with established RA on disease-modifying antirheumatic drug (DMARD) therapy compared with other clinical measures of remission.

We report a post hoc analysis of the Swedish Farmacotherapy (SWEFOT) randomised clinical trial in DMARD-naïve early RA (eRA), which featured an initial 3-month treatment with methotrexate (MTX) monotherapy. In patients whose disease did not respond to initial therapy, this was followed by a randomised comparison between nonbiological triple DMARD therapy and MTX plus biological (anti-tumour necrosis factor (TNF)) therapy. ${ }^{27} 28$ The MBDA score was measured in 
baseline serum samples from patients included in the SWEFOT clinical trial and studied as a predictor of RP after 1 year.

\section{METHODS}

\section{Study population}

This study was performed with data from the SWEFOT clinical trial, in which 487 DMARD-naïve patients with eRA (duration $<1$ year) from 15 different clinics in Sweden started 3 months of MTX treatment. After 3 months of MTX monotherapy, those whose disease did not respond (DAS28>3.2) were randomised into two groups: group A $(n=130)$ received MTX combined with sulfasalazine (SSZ) and hydroxychloroquine (HCQ) (triple therapy), and group B $(n=128)$ received MTX combined with infliximab. Approximately one-third of patients $(n=145)$ had a good response after 3 months of MTX monotherapy (DAS28 $\leq 3.2$ ) and they continued the treatment for 2 years. The trial was described in detail elsewhere. ${ }^{27}$

\section{Clinical and radiographic outcomes}

For this study complete sets of baseline demographic, serological and radiographic data, and clinical measures from 235 patients were analysed. Identification of clinical response to MTX monotherapy was done by using DAS28 based on ESR at 3-month follow-up ( $\leq 3.2$ : response; $>3.2$ : non-response). ${ }^{29}$ We also analysed CRP (mg/L), ESR and DAS28-CRP. The thresholds for disease activity levels according to these measures were as follows: for DAS28-ESR, low $\leq 3.2$, moderate 3.3-5.1 and high $>5.1^{30}$; for CRP, low $\leq 10 \mathrm{mg} / \mathrm{L}$, moderate $>10-30 \mathrm{mg} / \mathrm{L}$ and high $>30 \mathrm{mg} / \mathrm{L}^{31}$; and for DAS28-CRP, low $\leq 2.7$, moderate $2.8-4.1$ and high $>4.1 .^{32}$ Categorisation of patients in ESR low, moderate and high disease activity groups was done by using tertiles of the measure (for results based on continuous variables and tertiles using other disease activity measures, see online supplementary figures S1 and S2, respectively). X-rays of the hands and feet were done at baseline and after 1 year, and the van der Heijde modified Sharp score (SHS) was calculated. ${ }^{33}$ Patients whose SHS increased by more than five points from baseline to 1 year $(\Delta \mathrm{SHS}>5)$ were considered to have rapid RP (RRP). ${ }^{34} 35$ In addition, two other thresholds ( $\Delta \mathrm{SHS}>0$ and $\Delta \mathrm{SHS}>3$ ) were analysed for comparison. In the analyses of RRP that follow, these 235 patients were treated as a single group because their results define the overall outcome of the SWEFOT tight control strategy for patients with recent onset RA.

\section{Biomarker measurement and MBDA score}

The MBDA score was measured in baseline serum samples from the SWEFOT participants and is based on the following 12 biomarkers: vascular cell adhesion molecule 1, epidermal growth factor, vascular endothelial growth factor, interleukin 6, TNF receptor I, matrix metalloproteinases 1 and 3, bone glycoprotein 39 (YKL-40), leptin, resistin, serum amyloid A and CRP. These biomarkers were measured by electrochemiluminescencebased multiplexed immunoassays on the Meso Scale Discovery Multi-Array platform. ${ }^{36}$ The measured levels for each of the 12 biomarkers were weighted and combined using a validated formula to derive the MBDA score (Vectra DA score), which ranges from 1 to 100 . In this study, the following disease activity categories according to the MBDA score were used: low $(<30)$, moderate $(30-44)$ and high $(>44) .2223$

\section{Statistical analysis}

Descriptive statistics were prepared for demographics and baseline disease-related characteristics, including measures of disease activity. The association between RP at 1 year and each baseline disease activity measure was evaluated using univariate logistic regression. Wald's $\chi^{2}$ test $(\mathrm{p}<0.05)$ and the estimated OR and corresponding 95\% CI were used from the logistic model to assess the strength and direction of the association, respectively. Additionally, bivariate and multivariate logistic regression models were used to assess the association between RP and baseline MBDA score, while simultaneously accounting for potential confounders at baseline. For the multivariate analyses, we adjusted for all significant univariate predictors as in our recent report based on the same study populations. ${ }^{37}$ The reported $p$ values from these additional analyses were not adjusted for multiple testing. The difference in proportion of RP between patients with low/moderate and high MBDA score groups was compared by Fisher's Exact test. Probability plots were used to depict graphically the occurrence of RP over 1 year with patients stratified by the aforementioned baseline disease activity categories (low, moderate and high). Measures of sensitivity and specificity (positive predictive value and negative predictive value) were calculated to determine the degree to which the baseline MBDA score accurately predicts RP at 1 year.

\section{RESULTS}

\section{Description of the study cohort}

A total of 235 patients had complete radiographic, clinical and serological data for evaluation in this study ('study cohort'). Demographic and clinical data at baseline for these patients were similar to those for the overall SWEFOT trial population (table 1). Overall, the patients in the study cohort had a mean symptom duration of 6.1 months from diagnosis and moderate to high disease activity, as expected in an early-onset RA population.

Following 3 months of MTX therapy, 78 (33\%) of the 235 patients in the study cohort responded to treatment and continued to receive MTX monotherapy per protocol and 157 (67\%) did not respond and were randomised to receive triple DMARD therapy (group A) or MTX with infliximab (group B; table 2).

RRP, defined as $\Delta$ SHS $>5$ from baseline to 1 year, was observed for 43 of the 235 patients in the study cohort.

\section{Baseline characteristics and RP}

Among baseline parameters, MBDA, ESR and CRP values were significantly higher in patients with $\mathrm{RP}$ versus those without $(\mathrm{p}<0.001, \mathrm{p}=0.001$ and $\mathrm{p}=0.018$ respectively; table 1$)$. Mean changes in SHS from baseline to 1 year were 2.1 and 3.6 for the responder and non-responder groups, respectively and $13 \%$ of the responder group had RRP ( $\Delta$ SHS $>5$ ), compared with $21 \%$ in the non-responder group. Other thresholds including $\Delta \mathrm{SHS}>0$ and $\Delta \mathrm{SHS}>3$ were also tested (see online supplementary table $\mathrm{S} 1$ ).

\section{Relationship between RP and baseline level of MBDA score, CRP, ESR or DAS28}

The discriminative capacity of the baseline MBDA score, CRP, DAS28 and ESR for RP is illustrated by cumulative probability plots of $\triangle \mathrm{SHS}$ from baseline to 1 year (figure 1 and see online supplementary figures S1 and S2). The curve for the high MBDA group was markedly different from curves for the low or moderate MBDA groups (figure 1A). By contrast, curves for the three baseline CRP groups, two DAS28 groups and three ESR groups were more similar, with RRP being relatively frequent in all categories of these baseline measures (figure 1B-D, respectively). Mean $\Delta \mathrm{SHS}$ values and frequencies of progression for other thresholds of $\Delta$ SHS followed the same trends across categories of MBDA score as observed for $\Delta \mathrm{SHS}>5$ (table 2). 
Table 1 Baseline characteristics and demographic data of patients from SWEFOT trial

\begin{tabular}{|c|c|c|c|c|c|}
\hline \multirow[b]{2}{*}{$\begin{array}{l}\text { Baseline characteristics, } \\
\text { mean }( \pm \text { SD) }\end{array}$} & \multirow[b]{2}{*}{ All patients $(n=487)^{*}$} & \multicolumn{3}{|c|}{$\begin{array}{l}\text { Subset of patients with clinical measures at baseline and radiographs at } \\
\text { baseline and } 1 \text { year }\end{array}$} & \multirow[b]{2}{*}{ p Value } \\
\hline & & $\begin{array}{l}\text { Radiographic subset } \\
(\mathrm{n}=235)\end{array}$ & $\begin{array}{l}\text { Without progression } \\
(\Delta S H S \leq 5)(n=192)\end{array}$ & $\begin{array}{l}\text { With progression } \\
(\Delta S H S>5)(n=43)\end{array}$ & \\
\hline Female, $\mathrm{N}(\%)$ & $344(70)$ & $169(72)$ & $137(71)$ & $32(74)$ & 0.686 \\
\hline Symptom duration (months) & $6.2(4.57)$ & $6.1(5.1)$ & $6.0(5.38)$ & $6.6(3.61)$ & 0.502 \\
\hline Anti-CCP status, N (\%) & & & & & 0.075 \\
\hline Positive & $275(57)$ & $133(57)$ & $103(53)$ & $30(70)$ & \\
\hline Negative & $157(32)$ & $92(39)$ & $80(42)$ & $12(28)$ & \\
\hline Not available & $55(11)$ & $10(4)$ & $9(5)$ & $1(2)$ & \\
\hline RF status, N (\%) & & & & & 0.094 \\
\hline Positive & $330(68)$ & $153(65)$ & $120(63)$ & $33(77)$ & \\
\hline Negative & $152(31)$ & $80(34)$ & $70(36)$ & $10(23)$ & \\
\hline Not available & $5(1)$ & $2(1)$ & $2(1)$ & $0(0)$ & \\
\hline 28 swollen joint count & $10.8(5.28)$ & $10.8(5.31)$ & $10.7(5.30)$ & $11.0(5.43)$ & 0.807 \\
\hline 28 tender joint count & $9.6(6.07)$ & $9.3(5.86)$ & $9.4(5.99)$ & $8.77(5.25)$ & 0.518 \\
\hline ESR $(\mathrm{mm} / \mathrm{h})$ & $39.9(25.9)$ & $41.3(26.9)$ & $38.5(24.46)$ & $53.9(33.52)$ & 0.001 \\
\hline CRP level (mg/L) & $33.8(36.81)$ & $35.4(38.37)$ & $32.5(36.41)$ & $48.3(44.31)$ & 0.018 \\
\hline $\begin{array}{l}\text { Patient's global assessment of disease } \\
\text { activity (VAS 0-100 mm) score }\end{array}$ & $56(23.9)$ & $55.4(24.67)$ & $54.1(24.96)$ & $61.3(22.70)$ & 0.082 \\
\hline DAS28 & $5.7(1.01)$ & $5.7(1.02)$ & $5.7(1.00)$ & $5.9(1.14)$ & 0.107 \\
\hline DAS28-CRP & $6.5(1.22)$ & $5.4(0.99)$ & $5.3(0.97)$ & $5.5(1.04)$ & 0.237 \\
\hline MBDA score & $58.6(15.08)$ & $59.6(14.71)$ & $57.9(14.68)$ & $67.2(12.38)$ & $<0.001$ \\
\hline SHS mean (median) & $4.5(2)$ & $4.7(2)$ & $4.3(1)$ & $6.5(3)$ & 0.126 \\
\hline
\end{tabular}

\section{Discordance between MBDA scores and clinical} assessments: relationship to RP

As illustrated in figure 2, none of the patients had low DAS28-ESR or DAS28-CRP at baseline (because of the trial inclusion criteria), but among those with moderate DAS28-ESR/ CRP and low/moderate CRP, approximately 15\% developed RP during 1 year (figure $2 \mathrm{~A}-\mathrm{C}$, respectively). While all patients with low MBDA score had low CRP and no RP, a high MBDA score was observed in 59\% (42/71) of patients with low CRP and all rapid progression associated with low CRP $(n=10)$ occurred in the high MBDA subgroup (figure 2C). Thus, almost all patients with RP (42 of 43 cases) belonged to the high MBDA group $(\mathrm{n}=201)$ and represented $21 \%$ of that group versus only one case of progression (3.4\%) among patients with moderate $(n=29, \mathrm{p}=0.021)$, and none among patients with low MBDA score (figure 2D).

Table 2 Radiographic progression over 1 year stratified by clinical response at 3 months of MTX monotherapy

\begin{tabular}{|c|c|c|c|c|c|c|}
\hline & \multicolumn{2}{|c|}{$\Delta$ SHS from baseline } & \multirow{2}{*}{$\frac{\Delta S H S \leq 0}{n(\%)}$} & \multirow{2}{*}{$\frac{\Delta S H S>0}{n(\%)}$} & \multirow{2}{*}{$\frac{\Delta S H S>3}{n(\%)}$} & \multirow{2}{*}{$\frac{\Delta S H S>5}{n(\%)}$} \\
\hline & Mean $( \pm S D)$ & Median & & & & \\
\hline \multicolumn{7}{|l|}{ Baseline MBDA score } \\
\hline Low (MBDA <30, N=5) & $0.8(1.79)$ & 0 & $4(80)$ & $1(20)$ & $1(20)$ & 0 \\
\hline Moderate (MBDA 30-44, N=29) & $1.1(2.07)$ & 0 & $19(66)$ & $10(34)$ & $4(14)$ & $1(3)$ \\
\hline High (MBDA >44, N=201) & $3.4(6.44)$ & 1 & $92(46)$ & $109(54)$ & $67(33)$ & $42(21)$ \\
\hline \multicolumn{7}{|c|}{ Radiographic assessment at 1 year by response to MTX at 3 months* } \\
\hline Response to MTX (N=78) & $2.1(4.36)$ & 0 & $40(51)$ & $38(49)$ & $15(19)$ & $10(13)$ \\
\hline Non-response to MTX ( $\mathrm{N}=157)$ & $3.6(6.70)$ & 1 & $75(48)$ & $82(52)$ & $57(36)$ & $33(21)$ \\
\hline Group A $(\mathrm{N}=77) \dagger$ & $4.0(6.90)$ & 1 & $36(47)$ & $41(53)$ & $28(36)$ & $18(23)$ \\
\hline Group B $(N=75) \dagger$ & $3.2(6.71)$ & 0 & $38(51)$ & 37 (49) & $27(36)$ & $15(20)$ \\
\hline Total cohort $(\mathrm{N}=235)$ & $3.1(6.05)$ & 1 & $115(49)$ & $120(51)$ & $72(31)$ & $43(18)$ \\
\hline
\end{tabular}


A

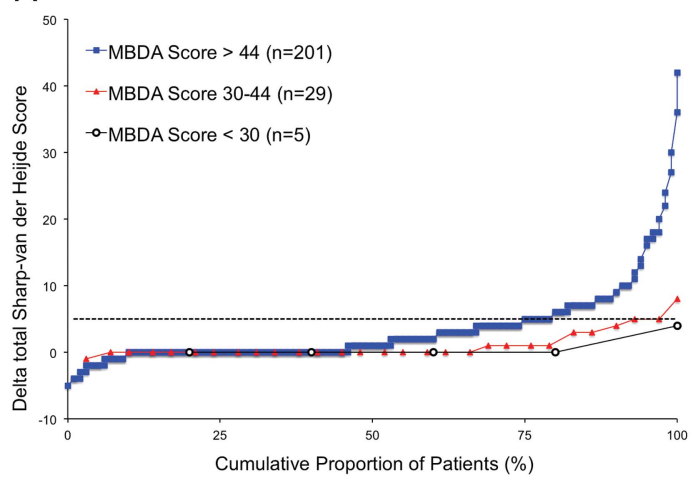

C

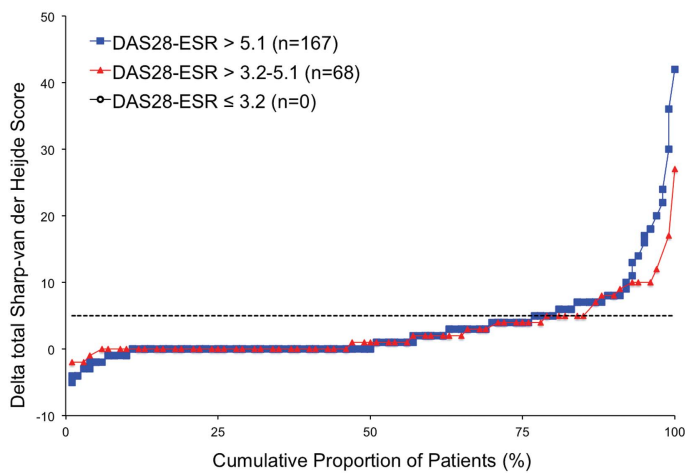

B

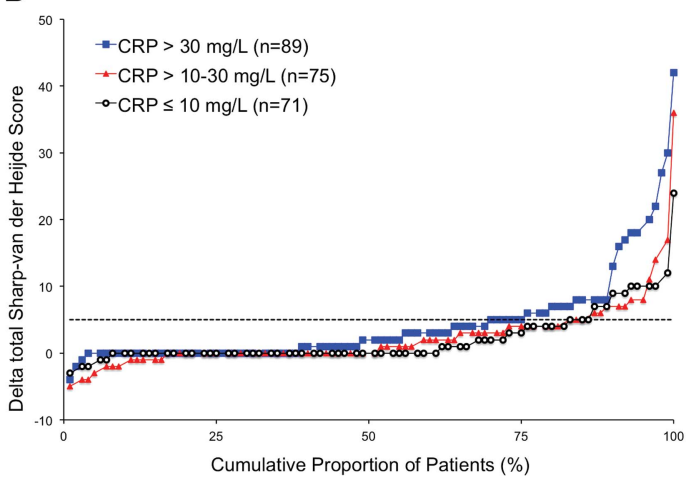

D

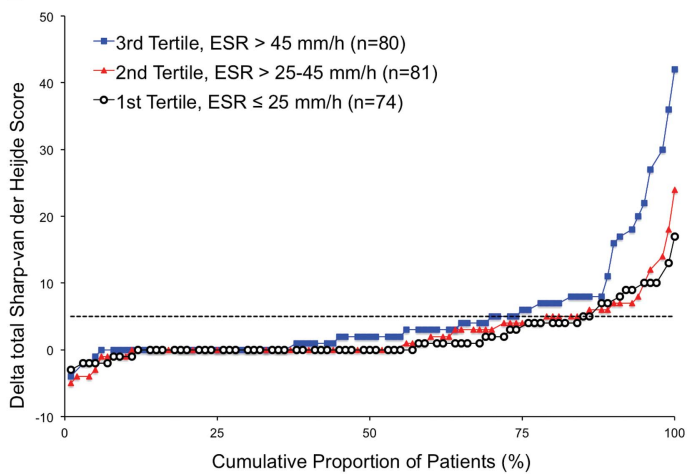

Figure 1 Probability plots of radiographic progression at year 1 for high, moderate and low disease activity patient $(\mathrm{N}=235)$ grouped according to baseline MBDA (A), CRP (B), DAS28 (C) and ESR (D). Each black circle represents a patient with low disease activity, red triangle-moderate disease activity and blue square- high disease activity. Horizontal dashed line represents $\triangle S H S=5$ from baseline to 1 year, above which the change is considered as rapid radiographic progression $(\triangle \mathrm{SHS}>5)$. DAS28, disease activity score; ESR, erythrocyte sedimentation rate; MBDA, multi-biomarker disease activity; SHS, Sharp-van der Heijde score.

The accuracy of the baseline MBDA score to predict RP at year 1 was assessed by calculating measures of sensitivity and specificity (see online supplementary table S2 and text S1). Additionally, the relationship between RP and baseline MBDA score was further examined in the subgroup of patients with high baseline scores (>44) (see online supplementary figures S3 and S4).

\section{Disease activity at baseline for predicting RRP}

Univariate analyses of the radiographic subgroup $(n=235)$ demonstrated significant associations with RRP, defined as $\triangle \mathrm{SHS}>5$ units in 1 year, for baseline MBDA score (the odds of $\mathrm{RP}$ increased by $5 \%$ for each 1 -unit increase in the MBDA score: $\mathrm{OR}=1.05, \mathrm{p}<0.001)$ and baseline CRP $(\mathrm{OR}=1.10$, $\mathrm{p}=0.018)$ but not for baseline DAS28-ESR $(\mathrm{OR}=1.31$, $\mathrm{p}=0.107)$ or DAS28-CRP $(\mathrm{OR}=1.22, \mathrm{p}=0.237) \quad($ table 3$)$. Further analyses of the high MBDA subset also confirmed that odds for RP is doubled in patients whose MBDA score is above 65 compared with those whose MBDA score is >44-65 (see online supplementary figure $\mathrm{S} 4$ ).

In bivariate analyses that adjusted the MBDA scores for 11 different clinical variables and for sex, one at a time, the baseline MBDA score was always an independent predictor of RRP (OR values: $1.04-1.06$, $\mathrm{p}$ values: 0.021 to $<0.001$ ).

Furthermore, MBDA score as a continuous variable was a strong independent predictor of RRP after 1 year $(\mathrm{OR}=1.05$, $\mathrm{p}<0.001$; table 3 ), using a multivariate logistic regression model with adjustment for all significant baseline predictors from univariate analyses (sex, symptom duration, current smoking status, erosions, Health Assessment Questionnaire score), as in our recent publication. ${ }^{37}$ When dichotomised into high MBDA score versus not, the adjusted OR for RRP after 1 year was 3.86 $(\mathrm{p}=0.04)$.

\section{DISCUSSION}

In this post hoc analysis of the SWEFOT trial we demonstrated that in DMARD-naïve patients with eRA, baseline serum levels of the 12-biomarker MBDA score may predict those that are at low versus relatively higher risk of RP $(0 \%, 3.4 \%$ and $21 \%$ RP among patients with low, moderate and high MBDA score, respectively). Our results also indicate that baseline MBDA scores discriminate risk for subsequent RP in SWEFOT more effectively than the baseline CRP or DAS28. Furthermore, MBDA score, both on a continuous and dichotomised (high vs low/moderate), scale was found to be an independent predictor of RP after adjustments for other predictors in this study population.

Early identification of patients with RA whose condition is likely to have a good or poor response to the treatment is very important for the optimal choice of the therapy. However, good clinical response does not guarantee good radiographic outcome. ${ }^{38-42}$ Therefore, predictors of clinical and radiographic response are vital for patients' long-term outcome.

We evaluated baseline MBDA score as a predictor of 1 year $\mathrm{RP}$, which was measured as the change in SHS. The definition of $\mathrm{RP}$ according to $\Delta \mathrm{SHS}$ varies in different clinical studies. Van der Helm-van Mil et $a l^{23}$ used $\Delta \mathrm{SHS}>3$ as the main definition for progression, though $\Delta \mathrm{SHS}>0$ and $\Delta \mathrm{SHS}>5$ were also applied for comparison. Vastesaeger et $a l^{34}$ tested different 


\begin{tabular}{|c|c|c|c|c|}
\hline \multicolumn{5}{|c|}{ A cross-classification of the MBDA score with DAS28-ESR at baseline } \\
\hline \multirow[b]{3}{*}{ Baseline MBDA } & \multicolumn{3}{|c|}{ Baseline DAS28-ESR } & \multirow[b]{3}{*}{ Total } \\
\hline & Low & Moderate & High & \\
\hline & $(\leq 3.2)$ & $(>3.2-5.1)$ & $(>5.1)$ & \\
\hline Low $(<30)$ & 0 & $0 / 3$ & $0 / 2$ & $0 / 5$ \\
\hline Moderate $(30$ - 44) & 0 & $1 / 14$ & $0 / 15$ & $1 / 29$ \\
\hline High (>44) & 0 & 9/51 & $33 / 150$ & $42 / 201$ \\
\hline Total & 0 & $10 / 68$ & $33 / 167$ & $43 / 235$ \\
\hline \multicolumn{5}{|c|}{ C cross-classification of the MBDA score with CRP at baseline } \\
\hline & \multicolumn{3}{|c|}{ Baseline Clinical CRP } & \\
\hline Baseline MBDA & $\begin{array}{l}\text { Low } \\
(\leq 10)\end{array}$ & $\begin{array}{l}\text { Moderate } \\
(>10-30)\end{array}$ & $\begin{array}{l}\text { High } \\
(>30)\end{array}$ & Total \\
\hline Low $(<30)$ & $0 / 5$ & 0 & 0 & $0 / 5$ \\
\hline Moderate (30 - 44) & $0 / 24$ & $0 / 4$ & $1 / 1$ & $1 / 29$ \\
\hline High (>44) & $10 / 42$ & $11 / 71$ & $21 / 88$ & $42 / 201$ \\
\hline Total & $10 / 71$ & $11 / 75$ & $22 / 89$ & $43 / 235$ \\
\hline
\end{tabular}
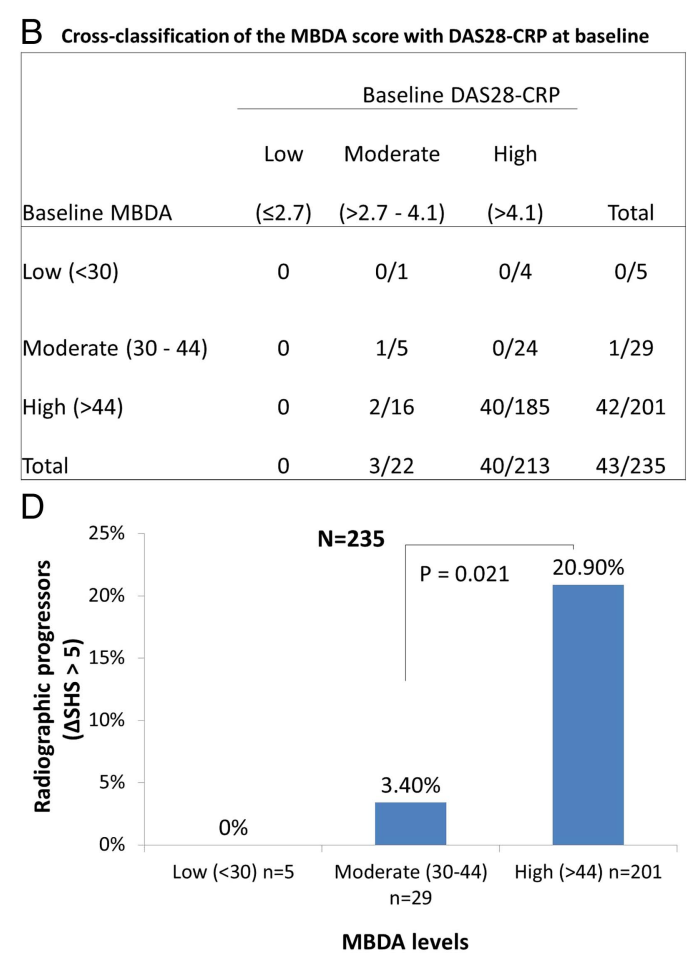

Figure 2 Cross tabulation of all analysed patients $(\mathrm{N}=235)$ and subset $(\mathrm{n}=43)$ with rapid radiographic progression $(\Delta S H S>5)$ over 1 year, by baseline disease activity measures. The denominator in each cell represents the number of patients cross classified by baseline MBDA score and DAS28-ESR (A), baseline MBDA score and DAS28-CRP (B) and baseline MBDA score and CRP (C) disease activity scores. The numerator in each cell represents the number of patients with radiographic progression at 1 year. (D) Radiographic progression for MBDA low, moderate and high score groups (\%). Radiographic progression at 1 year is defined by increase in SHS>5 compared with baseline. CRP, C-reactive protein; DAS28-CRP, disease activity score based on C-reactive protein; DAS28-ESR, disease activity score based on erythrocyte sedimentation rate; MBDA, multi-biomarker disease activity; SHS, Sharp-van der Heijde score.

threshold values for RP from $\Delta S H S>0$ to $\Delta S H S \geq 9$ and found that $\Delta$ SHS $\geq 5$ was a suitable definition for RRP. Bruynesteyn et $a l^{35}$ showed that $\Delta S H S \geq 5$ had $83 \%$ specificity for the smallest detectable difference in RP. Therefore we applied a threshold of $\Delta \mathrm{SHS}>5$ for RRP.

Previously, the MBDA score was evaluated and cut-offs were established for 'molecular remission' $(\leq 25)$, low $(<30)$, moderate $(30-44)$ and high $(>44)$ disease activity scores. ${ }^{22}$ As it was designed, the MBDA score is significantly associated with such disease activity measures as DAS28-CRP, DAS28-ESR, ESR, CRP, simple disease activity index and clinical disease activity index. 222526

The relationship between MBDA score and RP has been investigated in other settings. In the CAMERA trial, the MBDA score was predictive of RP with borderline significance after adjustment for rheumatoid factor and baseline erosions. ${ }^{25}$ In that study, baseline MBDA score was compared with RP over 2 years and $\Delta S H S>0$ was used as the cut-off. Perhaps most importantly, the sample size $(n=72)$ in that study was smaller. Van der Helm-van Mil et $a l^{23}$ showed that a greater proportion (93\%) of patients with MBDA $\leq 25$ ('molecular remission') had no progression $(\mathrm{SHS} \leq 3)$ compared with patients in DAS28-CRP $(<2.32 ; 80 \%)$ or American College of Rheumatology (ACR)/ European League Against Rheumatism (EULAR) (28 tender joint count, 28 swollen joint count, patient's global assessment and $\mathrm{CRP} \leq 1 ; 83 \%$ ) remission. Moreover, the difference in the proportion without progression was only significant $(p=0.001)$ for remission vs non-remission groups based on the MBDA score (but not when based on the DAS28-CRP or ACR/EULAR definitions of remission). Furthermore, their study showed that patients with high MBDA score were at sixfold higher risk of $\mathrm{RP}(\Delta \mathrm{SHS}>3)$ than those in MBDA remission. Finally, the proportion with RP in the DAS28-CRP remission group who had a high MBDA score (47\%) was twice as high as it was in all patients that met DAS28-CRP remission (20\%) criteria. $^{23}$ Although that study was based on the Leiden Early Arthritis Cohort, the samples were obtained at different time points during the disease course, while patients were already on established DMARD therapy, and it therefore conceptually addresses a different question compared with our study. Moreover, 20\% of patients from the former study, compared with only $2 \%$ from our study cohort, had remission or low disease activity by MBDA, consistent with the fact that the former were on stable DMARD therapy while patients in SWEFOT were DMARD naïve at inclusion. However, one important finding in these two studies is similar, namely that the MBDA score is a stronger predictor of 1-year RP than DAS28-CRP.

In the current study, patients with low or moderate MBDA score $(\leq 44)$ were shown to be at low risk of RP. Furthermore, when adjusted for commonly used markers and gender in bivariate and multivariate logistic regression analyses, the findings remained, indicating independence of the MBDA association with RP. We also demonstrated that MBDA score differentiated patients without progression from those with progression better than CRP. Sensitivity and specificity analysis revealed a strong negative prediction (radiographic non-progression). However, positive predictive value and specificity were very low, indicating that, though having relatively higher risk, the majority of patients with high MBDA score still did not progress radiographically over 1 year. These data suggest that baseline MBDA score might be used for identification of patients at 
Table 3 Univariate, bivariate and multivariate analyses of baseline MBDA score, DAS28 and CRP as predictors of 1-year radiographic progression

\begin{tabular}{|c|c|c|c|}
\hline & $\mathrm{OR}^{*}$ & $95 \% \mathrm{Cl}$ & p Valuet \\
\hline \multicolumn{4}{|l|}{ Univariate analyses } \\
\hline Baseline MBDA score & 1.05 & (1.02 to 1.08$)$ & $<0.001$ \\
\hline Baseline DAS28-ESR & 1.31 & (0.94 to 1.81$)$ & 0.107 \\
\hline Baseline DAS28-CRP & 1.22 & $(0.88$ to 1.71$)$ & 0.237 \\
\hline Baseline CRP (mg/L) & 1.10 & (1.02 to 1.18$)$ & 0.018 \\
\hline \multicolumn{4}{|l|}{ Bivariate models } \\
\hline Baseline MBDA adjusted for DAS28-ESR & 1.05 & (1.02 to 1.08$)$ & $<0.001$ \\
\hline Baseline MBDA adjusted for DAS28-CRP & 1.05 & (1.02 to 1.09$)$ & $<0.001$ \\
\hline Baseline MBDA adjusted for CRP & 1.06 & $(1.02$ to 1.10$)$ & 0.002 \\
\hline Baseline MBDA adjusted for ESR & 1.04 & (1.01 to 1.07$)$ & 0.021 \\
\hline Baseline MBDA adjusted for rheumatoid factor & 1.05 & (1.02 to 1.08$)$ & $<0.001$ \\
\hline Baseline MBDA adjusted for CCP status & 1.05 & (1.03 to 1.08$)$ & $<0.001$ \\
\hline Baseline MBDA adjusted for total swollen joint count & 1.05 & (1.02 to 1.08$)$ & $<0.001$ \\
\hline Baseline MBDA adjusted for total tender joint count & 1.05 & (1.02 to 1.08$)$ & $<0.001$ \\
\hline Baseline MBDA adjusted for global assessment of disease activity & 1.05 & (1.02 to 1.08$)$ & $<0.001$ \\
\hline Baseline MBDA adjusted for SHS & 1.05 & (1.02 to 1.08$)$ & $<0.001$ \\
\hline Baseline MBDA adjusted for symptom duration & 1.05 & (1.02 to 1.08$)$ & $<0.001$ \\
\hline Baseline MBDA adjusted for sex & 1.05 & (1.02 to 1.08$)$ & $<0.001$ \\
\hline \multicolumn{4}{|l|}{ Multivariate modelł } \\
\hline Baseline MBDA adjusted for sex, symptom duration, baseline erosions, current smoking status, HAQ score & 1.05 & $(1.02$ to 1.08$)$ & $<0.001$ \\
\hline High (>44) baseline MBDA score adjusted for sex, symptom duration, baseline erosions, current smoking status, HAQ score & 3.86 & (1.04 to 14.26$)$ & 0.04 \\
\hline
\end{tabular}

lower risk of progression and will help in appropriate choice of therapy for patients with a high MBDA score and at risk of RP.

Our study has some limitations. Since patients with low DAS28 were not included in the SWEFOT trial; it was not possible to analyse the predictive value of the MBDA score in this patient group. Also it should be noted that as a randomised control trial, the SWEFOT study does not fully represent the RA population. However, it was designed to be as close to a real-life eRA population as possible, with the only major inclusion criterion being DAS28 $>3.2$. The study is a post hoc analysis of the SWEFOT trial, which was designed primarily for comparison of biological and non-biological combination DMARD therapies. During the trial some patients had to switch from one drug to another for different reasons (lack of efficacy, side effects), and such switches could affect radiographic outcomes. However, any changes made in response to a lack of efficacy would most likely attenuate any true differences between the groups.

The strengths of this study were that it was based on a prospective, randomised trial, with a generous sample size, and that all the analyses presented here are based on the baseline clinical characteristics and the baseline MBDA score, information that could in principle be available to the clinician when making the first decision regarding therapy.

It will be important to study the predictive value of the MBDA score at additional time points for even longer follow-up times of clinical and radiographic data.

In conclusion, in DMARD-naïve patients with eRA, low/moderate MBDA score at baseline was shown to be associated with a very low risk of RP after 1 year. If confirmed in other studies, these data suggest that MBDA score can be useful in risk assessment for RP in eRA.

\section{Author affiliations}

${ }^{1}$ Unit of Clinical Therapy Research, Inflammatory Diseases (ClinTRID), Karolinska Institute, Stockholm, Sweden

${ }^{2}$ Crescendo Bioscience Inc., South San Francisco, California, USA

${ }^{3}$ Rheumatology Unit, Department of Medicine, Karolinska University Hospital and Karolinska Institute, Stockholm, Sweden

${ }^{4}$ Scott Cruickshank and Associates Inc., Santa Barbara, California, USA

${ }^{5}$ Section of Rheumatology, Institution of Clinical Sciences, University Hospital, Lund, Sweden

${ }^{6}$ Section of Rheumatology, Department of Medicine, Helsingborg Hospital, Helsingborg, Sweden

${ }^{7}$ Department of Orthopaedics, Institution of Clinical Sciences, Lund University, Lund, Sweden

Correction notice This article has been corrected twice since it was published Online First. Minor changes have been made to the Results section of the Abstract. In addition, a minor correction has been made to a value in table 1 .

Acknowledgements The SWEFOT Trial Investigators Group, besides the authors: Johan Bratt, Stockholm; Kristina Albertsson, Stockholm; Lars Cöster, Linköping; Eva Waltbrand, Borås; Agneta Zickert, Stockholm; Jan Theander, Kristianstad; Åke Thörner, Eskilstuna; Helena Hellström, Falun; Annika Teleman, Halmstad; Christina Dackhammar, Mölndal; Finn Akre, Örebro; Lotta Ljung, Umeå; Rolf Oding, Västerås; Katerina Chatzidionysiou, Stockholm; Margareta Wörnert, Stockholm. We would like to thank all participating patients and the study nurses, co-investigators and colleagues who made this trial possible.

Contributors $\mathrm{KH}$ had the lead role in management of the merged clinical and serological datasets and analyses thereof, discussions with collaborators, drafting of the manuscript and its final approval for submission. SS contributed to the management of the merged clinical and serological datasets and had a lead role in the statistical analyses thereof, participated in discussions with collaborators, critically 
reviewed the manuscript and approved the final version for submission. RB, DC, ES and $\mathrm{SC}$ were responsible for the serum analyses and the MBDA score generation, quality control of these data, and contributed to analysis and interpretations of these data. They all reviewed the manuscript critically and approved the final version for submission. KF had the main responsibility of the radiological scoring in the SWEFOT trial and supported the statistical analyses and interpretations thereof, participated in discussions with collaborators, critically reviewed the manuscript and approved the final version for submission. IP and PG were key investigators and steering committee members in the SWEFOT trial. They supported the statistical analyses and interpretations of the data in the current study, participated in discussions with collaborators, critically reviewed the manuscript and approved the final version for submission. RvV was an investigator in the SWEFOT trial and responsible investigator of the study reported here. He designed the study and organised the collaboration with Crescendo Biosciences Inc for the patients' serum analyses and the MBDA score generation. He also discussed and reviewed the manuscript critically and gave his final approval for submission of the manuscript.

Funding The study was supported in part by a grant from the Swedish Rheumatism Association. Some of the authors were supported by clinical research funds from Stockholm County (ALF funds). An annual unrestricted grant was provided by Schering-Plough Sweden that was used to support a study coordinator and a medical monitor for the original clinical trial. The analyses of the MBDA score were done by Crescendo Bioscience Inc (South San Francisco, California, USA), at no cost to the investigators. No financial support was provided by Crescendo Bioscience Inc or other companies for this study.

Competing interests RvV received research support and/or honoraria from AbbVie, Biotest, BMS, GSK, Lilly, Merck, Pfizer, Roche, UCB, Vertex. IP received research funding and/or honoraria from AbbVie, UCB Pharma and Pfizer, not related to this study. ES is an employee of and holds stock options from Crescendo Bioscienc Inc. DC receives consulting fees and stock options from Crescendo Bioscience Inc. KF has received honoraria from AbbVie and BMS, not related to this study. RB is an employee of and receives stock options form Crescendo Bioscience Inc. SC is an independent contractor to Crescendo Bioscience In and is paid on an hourly basis. KH, SS and PG: none declared.

Ethics approval This study was approved by the regional ethics committees of all participating units.

Provenance and peer review Not commissioned; externally peer reviewed.

Open Access This is an Open Access article distributed in accordance with the Creative Commons Attribution Non Commercial (CC BY-NC 3.0) license, which permits others to distribute, remix, adapt, build upon this work non-commercially, and license their derivative works on different terms, provided the original work is properly cited and the use is non-commercial. See: http://creativecommons.org/ licenses/by-nc/3.0/

\section{REFERENCES}

1 Mclnnes IB, Schett G. The pathogenesis of rheumatoid arthritis. N Engl J Med 2011;365:2205-19.

2 Uhlig T, Kvien TK. Is rheumatoid arthritis really getting less severe? Nat Rev Rheumatol 2009:5:461-4

3 Liao KP, Weinblatt ME, Cui J, et al. Clinical predictors of erosion-free status in rheumatoid arthritis: a prospective cohort study. Rheumatology (Oxford) 2011;50:1473-9.

4 Smolen JS, Van Der Heijde DM, St Clair EW, et al. Predictors of joint damage in patients with early rheumatoid arthritis treated with high-dose methotrexate with or without concomitant infliximab: results from the ASPIRE trial. Arthritis Rheum 2006:54:702-10.

5 Garnero P, Landewe R, Boers $M$, et al. Association of baseline levels of markers of bone and cartilage degradation with long-term progression of joint damage in patients with early rheumatoid arthritis - the COBRA Study. Arthritis Rheum 2002:46:2847-56.

6 Quinn MA, Gough AKS, Green MJ, et al. Anti-CCP antibodies measured at disease onset help identify seronegative rheumatoid arthritis and predict radiological and functional outcome. Rheumatology (Oxford) 2006;45:478-80.

7 Goronzy JJ, Matteson EL, Fulbright JW, et al. Prognostic markers of radiographic progression in early rheumatoid arthritis. Arthritis Rheum 2004;50:43-54.

8 Avouac J, Gossec L, Dougados M. Diagnostic and predictive value of anti-cyclic citrullinated protein antibodies in rheumatoid arthritis: a systematic literature review. Ann Rheum Dis 2006;65:845-51.

9 Nell VPK, Machold KP, Stamm TA, et al. Autoantibody profiling as early diagnostic and prognostic tool for rheumatoid arthritis. Ann Rheum Dis 2005;64:1731-6.

10 Forslind $\mathrm{K}$, Ahlmen M, Eberhardt $\mathrm{K}$, et al. Prediction of radiological outcome in early rheumatoid arthritis in clinical practice: role of antibodies to citrullinated peptides (anti-CCP). Ann Rheum Dis 2004;63:1090-5.

11 Meyer 0 , Nicaise-Roland P, Santos MD, et al. Serial determination of cyclic citrullinated peptide autoantibodies predicted five-year radiological outcomes in a prospective cohort of patients with early rheumatoid arthritis. Arthritis Res Ther 2006:8:R40.

12 Saag KG, Cerhan JR, Kolluri $S$, et al. Cigarette smoking and rheumatoid arthritis severity. Ann Rheum Dis 1997:56:463-9.

13 Masdottir B, Jonsson T, Manfredsdottir V, et al. Smoking, rheumatoid factor isotypes and severity of rheumatoid arthritis. Rheumatology (Oxford) 2000;39:1202-5

14 Visser K, Goekoop-Ruiterman YP, de Vries-Bouwstra JK, et al. A matrix risk model for the prediction of rapid radiographic progression in patients with rheumatoid arthritis receiving different dynamic treatment strategies: post hoc analyses from the BeSt study. Ann Rheum Dis 2010;69:1333-7.

15 Bakker MF, Verstappen SM, Welsing PM, et al. The relation between cartilage biomarkers (C2C, C1, 2C, CS846, and CPII) and the long-term outcome of rheumatoid arthritis patients within the CAMERA trial. Arthritis Res Ther 2011;13: R70.

16 Niki Y, Takeuchi T, Nakayama M, et al. Clinical significance of cartilage biomarkers for monitoring structural joint damage in rheumatoid arthritis patients treated with anti-TNF therapy. Plos One 2012;7:e37447.

17 Andersson ML, Svensson B, Petersson IF, et al. Early increase in serum-COMP is associated with joint damage progression over the first five years in patients with rheumatoid arthritis. BMC Musculoskelet Disord 2013;14:229.

18 Rho YH, Solus J, Sokka T, et al. Adipocytokines are associated with radiographic joint damage in rheumatoid arthritis. Arthritis Rheum 2009;60: 1906-14.

19 Syversen SW, Goll GL, Haavardsholm EA, et al. A high serum level of eotaxin (CCL 11) is associated with less radiographic progression in early rheumatoid arthritis patients. Arthritis Res Ther 2008;10:R28.

20 Ortea I, Roschitzki B, Ovalles JG, et al. Discovery of serum proteomic biomarkers for prediction of response to infliximab (a monoclonal anti-TNF antibody) treatment in rheumatoid arthritis: an exploratory analysis. J Proteomics 2012;77:

372-82.

21 Wagner $\mathrm{C}$, Chen D, Fan $\mathrm{H}$, et al. Evaluation of serum biomarkers associated with radiographic progression in methotrexate-naive rheumatoid arthritis patients treated with methotrexate or golimumab. J Rheumatol 2013;40:590-8.

22 Curtis JR, van der Helm-van Mil AH, Knevel $R$, et al. Validation of a novel multibiomarker test to assess rheumatoid arthritis disease activity. Arthritis Care Res 2012:64:1794-803

23 van der Helm-van Mil AH, Knevel R, Cavet G, et al. An evaluation of molecular and clinical remission in rheumatoid arthritis by assessing radiographic progression. Rheumatology (Oxford) 2013;52:839-46.

24 Centola M, Cavet G, Shen $Y$, et al. Development of a multi-biomarker disease activity test for rheumatoid arthritis. PloS One 2013;8:e60635.

25 Bakker MF, Cavet G, Jacobs JW, et al. Performance of a multi-biomarker score measuring rheumatoid arthritis disease activity in the CAMERA tight control study. Ann Rheum Dis 2012;71:1692-7.

26 Hirata S, Dirven L, Shen $Y$, et al. A multi-biomarker score measures rheumatoid arthritis disease activity in the BeSt study. Rheumatology (Oxford) 2013;52: 1202-7.

27 van Vollenhoven RF, Ernestam S, Geborek P, et al. Addition of infliximab compared with addition of sulfasalazine and hydroxychloroquine to methotrexate in patients with early rheumatoid arthritis (SWEFOT trial): 1-year results of a randomised trial. Lancet 2009:374:459-66.

28 van Vollenhoven RF, Geborek P, Forslind K, et al. Conventional combination treatment versus biological treatment in methotrexate-refractory early rheumatoid arthritis: 2 year follow-up of the randomised, non-blinded, parallel-group SWEFOT trial. Lancet 2012;379:1712-20.

29 Prevoo ML, van't Hof MA, Kuper HH, et al. Modified disease activity scores that include twenty-eight-joint counts. Development and validation in a prospective longitudinal study of patients with rheumatoid arthritis. Arthritis Rheum 1995;38:44-8.

30 Van Gestel AM, Prevoo ML, van't Hof MA, et al. Development and validation of the European League Against Rheumatism response criteria for rheumatoid arthritis. Comparison with the preliminary American College of Rheumatology and the World Health Organization/International League Against Rheumatism Criteria. Arthritis Rheum 1996;39:34-40.

31 Takahashi N, Kojima T, Kaneko A, et al. Clinical efficacy of abatacept compared to adalimumab and tocilizumab in rheumatoid arthritis patients with high disease activity. Clin Rheumatol 2013;33:39-47.

32 Inoue $\mathrm{E}$, Yamanaka $\mathrm{H}$, Hara $\mathrm{M}$, et al. Comparison of Disease Activity Score (DAS) 28- erythrocyte sedimentation rate and DAS28- C-reactive protein threshold values. Ann Rheum Dis 2007;66:407-9.

33 van der Heijde D. How to read radiographs according to the Sharp/van der Heijde method. J Rheumatol 2000;27:261-3.

34 Vastesaeger $\mathrm{N}, \mathrm{Xu}$ S, Aletaha D, et al. A pilot risk model for the prediction of rapid radiographic progression in rheumatoid arthritis. Rheumatology (Oxford) 2009;48:1114-21

35 Bruynesteyn $K$, van der Heijde $D$, Boers $M$, et al. Determination of the minimal clinically important difference in rheumatoid arthritis joint damage of the Sharp/van 
der Heijde and Larsen/Scott scoring methods by clinical experts and comparison with the smallest detectable difference. Arthritis Rheum 2002;46:913-20.

36 Eastman PS, Manning WC, Qureshi F, et al. Characterization of a multiplex, 12-biomarker test for rheumatoid arthritis. J Pharm Biome Anal 2012;70: $415-24$.

37 Saevarsdottir S, Rezaei $\mathrm{H}$, Geborek P, et al. Current smoking status is a strong predictor of RP in early rheumatoid arthritis: results from the SWEFOT trial. Ann Rheum Dis Published Online First: 15 March 2014. doi:10.1136/annrheumdis2013-204601.

38 Molenaar ET, Voskuyl AE, Dinant HJ, et al. Progression of radiologic damage in patients with rheumatoid arthritis in clinical remission. Arthritis Rheum 2004:50:36-42.

39 Rezaei H, Saevarsdottir S, Forslind $\mathrm{K}$, et al. In early rheumatoid arthritis, patients with a good initial response to methotrexate have excellent 2-year clinical outcomes, but radiological progression is not fully prevented: data from the methotrexate responders population in the SWEFOT trial. Ann Rheum Dis 2012;71:186-91.

40 Landewe $\mathrm{R}$, Geusens $\mathrm{P}$, Boers $\mathrm{M}$, et al. Markers for type II collagen breakdown predict the effect of disease-modifying treatment on long-term radiographic progression in patients with rheumatoid arthritis. Arthritis Rheum 2004;50: 1390-9.

41 Lillegraven S, Prince FH, Shadick NA, et al. Remission and radiographic outcome in rheumatoid arthritis: application of the 2011 ACR/EULAR remission criteria in an observational cohort. Ann Rheum Dis 2012;71:681-6.

42 Klarenbeek NB, Koevoets R, van der Heijde DM, et al. Association with joint damage and physical functioning of nine composite indices and the 2011 ACR/ EULAR remission criteria in rheumatoid arthritis. Ann Rheum Dis 2011;70: $1815-21$. 